\title{
Research on Lateral-Directional Dynamic Characteristics and Control for Large Envelope Flight of Modular Aircraft
}

\author{
Wen-Xin ZHANGa ${ }^{\mathrm{a}}$, Li-Xin WANG ${ }^{\mathrm{b}}$ and Ting YUE ${ }^{\mathrm{c}, *}$ \\ School of Aeronautic Science and Engineering, Beihang University, Beijing 100191, China \\ aStanley_Z@126.com, bwlx_c818@163.com, cyueting_buaa@163.com \\ ${ }^{*}$ Corresponding author
}

Keywords: Modular aircraft, Large envelope flight, LPV, Dynamic characteristics, Self-scheduled parameter adjustment.

\begin{abstract}
The linear parameter varying (LPV) system model of the lateral-directional dynamics for large envelope flight of modular aircraft including various configurations was built. The validity of the aircraft LPV model was verified by the comparison of dynamic simulation between the LPV model and the nonlinear model. The lateral-directional dynamic characteristics under different configurations of modular aircraft in large flight envelope were studied. The stability augmentation system (SAS) for the inner loop and the self-scheduled parameter adjusting $H_{\infty}$ robust controller for the outer loop of the control system were designed based on the lateral-directional dynamic LPV model of modular aircraft to guarantee the control effect for aircraft lateral-directional motion. The closed loop system was simulated and the simulation results indicate the integrated control system could achieve precision tracking of modular aircraft lateral-directional command signals under different configurations in large flight envelope.
\end{abstract}

\section{Introduction}

Modular aircraft is considered to be the multi-role unmanned aerial vehicle (UAV) that could satisfy future demands. It consists of several demountable structural modules and employs compatible modules to accomplish different military missions, which improves operational efficiency of the aircraft conveniently and swiftly.

Due to different aircraft configurations caused by different wing modules, the dynamic characteristics of the aircraft will change substantially, and there will also be a substantial change in aircraft stability and manoeuvrability. In addition, the flight envelope of modular aircraft is larger than the single configuration aircraft. The aircraft stability and manoeuvrability will change greatly as the flight velocity and altitude change. As a result, it is of significance and necessity to analyze the dynamic characteristics of modular aircraft under its different configurations in the large flight envelope.

The lateral-directional dynamic characteristics of modular aircraft vary substantially under different configurations, and the flight envelope gets larger. The classic control method designs particular controllers at multiple working points and then employs gain-scheduled methods in the flight envelope according to the flight parameter to obtain the final controller. But when it comes to modular aircraft, the controller should change according to not merely the flight parameter, but the configuration as well. In this situation, using the classic control method would result in very tedious work when we design modular aircraft lateral-directional controller, nor would it guarantee robustness of the system. So the classic control method could not satisfy the control demand for modular aircraft. As a result, it becomes an urgent problem to design the flight control system for modular aircraft in large flight envelope to realize the flight control objectives.

One practicable way is to describe the dynamic equations of the modular aircraft by a LPV model, and then employ self-scheduled parameter adjusting control methods based on LPV model to implement the control [1-4]. The merit of this method is it treats the gain-scheduled controller as an integral. Because the parameter-dependent controller would make self-scheduled adjustment, the 
design of tedious planning and scheduling could be eliminated [5].

In this paper, the research object is the lateral-directional motion of modular aircraft. The lateraldirectional dynamic LPV model of modular aircraft is built. And the modular aircraft lateraldirectional dynamic characteristics under different configurations in large flight envelope are mainly studied. According to the features of modular aircraft, the self-scheduled parameter adjusting control method is designed for the aircraft lateral-directional control system based on the LPV model of it. Simulation is implemented to verify the controller's ability to realize precision tracking of modular aircraft lateral-directional command signals in large flight envelope.

\section{Features of Modular Aircraft}

Modular aircraft has demountable wings. The high-aspect-ratio straight wing is used under longendurance configuration for surveillance missions, while the low-aspect-ratio swept wing is used under high-speed configuration for strike missions. The stability and manoeuvrability of modular aircraft will change substantially due to the change of the configuration, because the wing area, sweep angle, area and position of control surface and other factors will be different under different configurations, affecting aircraft dynamic characteristics.

Modular aircraft has a wider range of flight envelope compared to fixed configuration aircraft because of its multiple configurations. Because aircraft aerodynamic derivatives vary according to the change of flight velocity and altitude, there will be a bigger change in the stability and manoeuvrability of modular aircraft in its flight envelope.

The schematic diagram of the two configurations of modular aircraft is shown in Fig. 1 and the flight envelope is shown in Fig. 2.
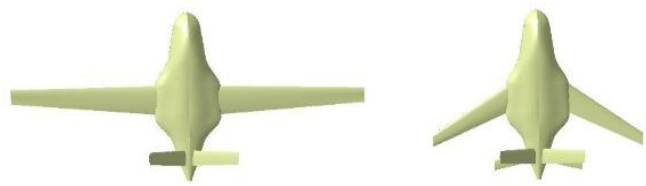

Fig. 1. Schematic diagram of different configurations of modular aircraft.

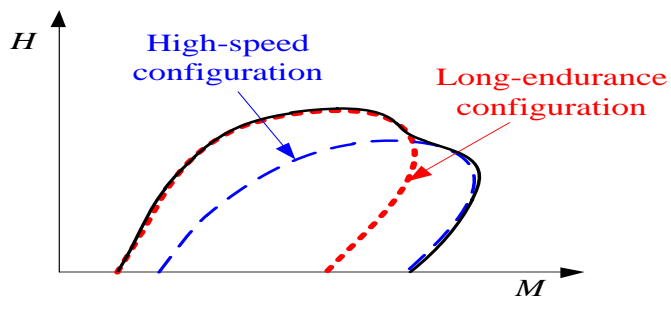

Fig. 2. Schematic diagram of the flight envelope of modular aircraft.

\section{Lateral-Directional LPV Model}

The LPV system is described by the following equations:

$$
\left\{\begin{array}{l}
\dot{\mathbf{x}}(t)=\mathbf{A}(\boldsymbol{\theta}) \mathbf{x}(t)+\mathbf{B}(\boldsymbol{\theta}) \mathbf{u}(t) \\
\mathbf{y}(t)=\mathbf{C}(\boldsymbol{\theta}) \mathbf{x}(t)+\mathbf{D}(\boldsymbol{\theta}) \mathbf{u}(t)
\end{array}\right.
$$

where $\boldsymbol{\theta}$ is a time-varying parameter vector, which consists of system outputs and inputs. State matrixes $\mathbf{A}, \mathbf{B}, \mathbf{C}, \mathbf{D}$ are functions of fixed matrixes of $\boldsymbol{\theta}$.

The dynamic system of the flight vehicle is complex, and there is a complicated function relationship between the aerodynamic force of the aircraft, the shape of the aircraft, the state parameters and other factors. In order to simplify the situation, we make following assumptions when deduce the equation of motion [6,7]: The earth is flat and the curvature and rotation of the earth are neglected. The flight vehicle is rigid and the elastic deformation and rotating parts of the 
body are neglected. The atmosphere is static and standard and the wind effects are neglected.

The aircraft reference motion is assumed as symmetric stationary straight flight. The perturbation motion caused by control surface and other factors is a small quantity compared to the reference motion. Based on the linear small-perturbation theory, the modular aircraft lateral-directional smallperturbation linear mathematical model can be expressed as:

$$
\begin{aligned}
& \frac{d}{d t}\left[\begin{array}{c}
\beta \\
p \\
r \\
\phi
\end{array}\right]=\left[\begin{array}{cccc}
\bar{Y}_{\beta} & \alpha_{*}+\bar{Y}_{p} & \bar{Y}_{r}-1 & g \cos \theta_{*} / V_{*} \\
\bar{L}_{\beta} & \bar{L}_{p} & \bar{L}_{r} & 0 \\
\bar{N}_{\beta} & \bar{N}_{p} & \bar{N}_{r} & 0 \\
0 & 1 & \tan \theta_{*} & 0
\end{array}\right]\left[\begin{array}{l}
\beta \\
p \\
r \\
\phi
\end{array}\right] \\
& +\left[\begin{array}{cc}
0 & \bar{Y}_{\delta_{r}} \\
\bar{L}_{\delta_{a}} & \bar{L}_{\delta_{r}} \\
\bar{L}_{\delta_{r}} & \bar{N}_{\delta_{r}} \\
0 & 0
\end{array}\right]\left[\begin{array}{l}
\delta_{a} \\
\delta_{r}
\end{array}\right] .
\end{aligned}
$$

where

$$
\begin{aligned}
& \bar{Y}_{i}=\frac{Y_{i}}{m V_{*}}, i \in\left\{\beta, p, r, \delta_{r}\right\}, \\
& \bar{L}_{i}=\frac{L_{i}+\left(I_{z x} / I_{z}\right) N_{i}}{I_{x}-I_{z x}^{2} / I_{x}}, \bar{N}_{i}=\frac{N_{i}+\left(I_{z x} / I_{z}\right) L_{i}}{I_{z}-I_{z x}^{2} / I_{x}}, \\
& i \in\left\{\beta, p, r, \delta_{a}, \delta_{r}\right\} .
\end{aligned}
$$

According to (2), in the situation that the reference motion is symmetric stationary straight flight, modular aircraft lateral-directional dynamic model can be represented in matrix form:

$$
\dot{\mathbf{x}}=\mathbf{A} \mathbf{x}+\mathbf{B u} .
$$

where $\mathbf{x}$ is the state vector. $\mathbf{A}$ is the state matrix. $\mathbf{B}$ is the control matrix and $\mathbf{u}$ is the control input.

Modular aircraft aerodynamic derivatives vary at different state points in the flight envelope because of the change of the configuration and flight velocity and altitude. Furthermore, it results in the variation of matrix elements in $\mathbf{A}$ and $\mathbf{B}$ in (3). The matrixes $\mathbf{A}$ and $\mathbf{B}$ in modular aircraft lateral-directional dynamic model have a function relationship with flight velocity $V$ and altitude $H$ and the aircraft configuration $C$. Based on that fact, we build the modular aircraft lateral-directional LPV model in large flight envelope:

$$
\dot{\mathbf{x}}=\mathbf{A}(\boldsymbol{\theta}) \mathbf{x}+\mathbf{B}(\boldsymbol{\theta}) \mathbf{u}
$$

where

$$
\begin{gathered}
\mathbf{A}(\boldsymbol{\theta})=\left[\begin{array}{cccc}
\bar{Y}_{\beta}(\boldsymbol{\theta}) & \alpha_{*}+\bar{Y}_{p}(\boldsymbol{\theta}) & \bar{Y}_{r}(\boldsymbol{\theta})-1 & g \cos \theta_{*} / V_{*} \\
\bar{L}_{\beta}(\boldsymbol{\theta}) & \bar{L}_{p}(\boldsymbol{\theta}) & \bar{L}_{r}(\boldsymbol{\theta}) & 0 \\
\bar{N}_{\beta}(\boldsymbol{\theta}) & \bar{N}_{p}(\boldsymbol{\theta}) & \bar{N}_{r}(\boldsymbol{\theta}) & 0 \\
0 & 1 & \tan \theta_{*} & 0
\end{array}\right], \\
\mathbf{B}(\boldsymbol{\theta})=\left[\begin{array}{cc}
0 & \bar{Y}_{\delta_{r}}(\boldsymbol{\theta}) \\
\bar{L}_{\delta_{a}}(\boldsymbol{\theta}) & \bar{L}_{\delta_{r}}(\boldsymbol{\theta}) \\
\bar{L}_{\delta_{r}}(\boldsymbol{\theta}) & \bar{N}_{\delta_{r}}(\boldsymbol{\theta}) \\
0 & 0
\end{array}\right], \mathbf{x}=\left[\begin{array}{c}
\beta \\
p \\
r \\
\phi
\end{array}\right], \mathbf{u}=\left[\begin{array}{c}
\delta_{a} \\
\delta_{r}
\end{array}\right], \\
\boldsymbol{\theta}=(M a, H, C), M a \in[0.2,0.6], H \in[3000,10000], C=1,2 .
\end{gathered}
$$


when $C=1$, the aircraft is under the long-endurance configuration and when $C=2$, it is under the high-speed configuration.

When we build modular aircraft lateral-directional LPV model, the variability of the LPV model must be verified. We realize the verification by comparing the simulation results between the LPV model and nonlinear model. The surface command is given to the aircraft LPV model and nonlinear model at certain state point. The comparison of responses of aircraft motion parameters is shown in Fig. 3. ( $M a=0.2, H=3 \mathrm{~km}, 5^{\circ}$ step input of aileron)
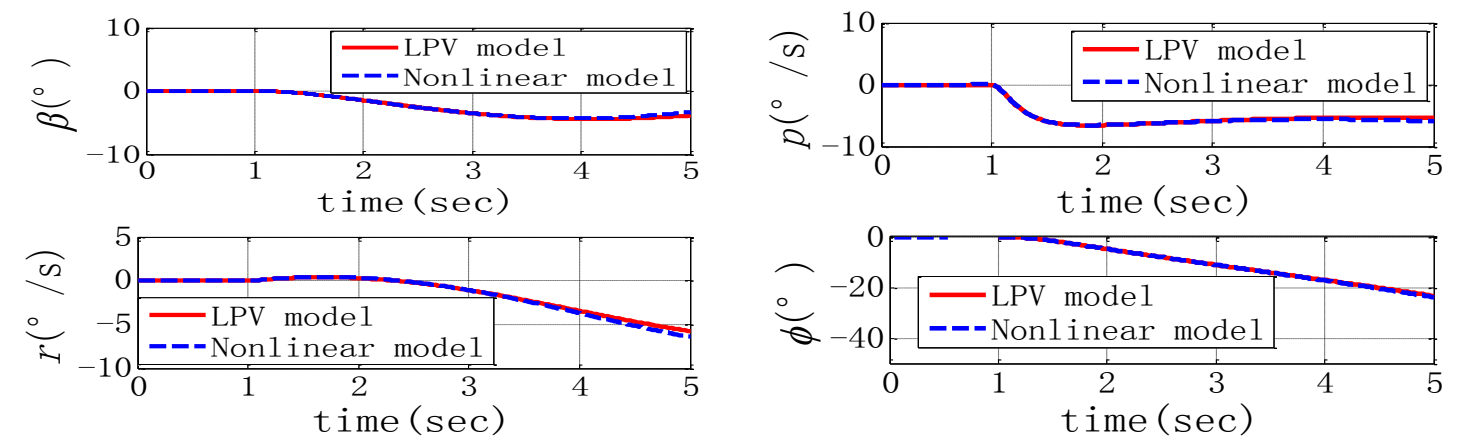

Fig. 3. Comparison of responses between LPV model and nonlinear model

The results show that responses to a surface command between the LPV model and nonlinear model are close, indicating the LPV model has variability.

When we design the gain-scheduled controller based on a LPV model, each controller at certain state point for the linear time invariant (LTI) system can be designed according to fixed parameter vectors. The final controller depends on the parameter vector $\boldsymbol{\theta}$. The mathematical form of the controller can be expressed as following [8]:

$$
\left\{\begin{array}{l}
\dot{\mathbf{x}}_{k}=\mathbf{A}_{k}(\boldsymbol{\theta}) \mathbf{x}_{k}+\mathbf{B}_{k}(\boldsymbol{\theta}) \mathbf{y} \\
\mathbf{u}=\mathbf{C}_{k}(\boldsymbol{\theta}) \mathbf{x}_{k}+\mathbf{D}_{k}(\boldsymbol{\theta}) \mathbf{y}
\end{array} .\right.
$$

The gain-scheduled controller for the LPV system has a function relationship with the variable gain parameter $\boldsymbol{\theta}$. For general system control problems, the gain scheduled controller should stabilize the closed loop system and meet the control performance [9-11]. The problem can be done by solving a group of linear matrix inequalities (LMI) based on convex optimization theory [12-14].

\section{Analysis of Lateral-Directional Dynamic Characteristics}

We choose modular aircraft's flight conditions under its different configurations (long-endurance and high-speed configuration) at typical state points $(0.2 \mathrm{Ma}, 3 \mathrm{~km}$ and $0.6 \mathrm{Ma}, 10 \mathrm{~km}$.) to analyze the lateral-directional dynamic characteristics. The important lateral-directional derivatives of modular aircraft at different state points are shown in Table 1. (1 refers to long-endurance configuration at state point of $0.2 \mathrm{Ma}, 3 \mathrm{~km}$. 2 refers to high-speed configuration at state point of $0.2 \mathrm{Ma}, 3 \mathrm{~km} .3$ refers to long-endurance configuration at state point of $0.6 \mathrm{Ma}, 10 \mathrm{~km} .4$ refers to high-speed configuration at state point of $0.6 \mathrm{Ma}, 10 \mathrm{~km}$.)

Table 1. Lateral-directional derivatives (unit: $\left({ }^{\circ}\right)^{-1}$ )

\begin{tabular}{|c|c|c|c|c|c|c|}
\hline State & $C_{\mathrm{lp}}$ & $C_{\mathrm{nr}}$ & $C_{\mathrm{l} \beta}$ & $C_{\mathrm{n} \beta}$ & $C_{\mathrm{l} \delta \mathrm{a}}$ & $C_{\mathrm{n} \delta \mathrm{r}}$ \\
\hline 1 & -0.64 & -0.02 & -0.05 & 0.06 & -0.22 & -0.04 \\
\hline 2 & -0.55 & -0.09 & -0.12 & 0.14 & -0.15 & -0.09 \\
\hline 3 & -0.73 & -0.03 & -0.06 & 0.07 & -0.25 & -0.04 \\
\hline 4 & -0.60 & -0.10 & -0.14 & 0.16 & -0.17 & -0.10 \\
\hline
\end{tabular}

Table 1 shows the change of the aircraft configuration will lead to variation in lateral-directional 
dimensionless derivatives (such as the roll damping derivative $C_{\mathrm{lp}}$, yaw damping derivative $C_{\mathrm{nr}}$, dihedral derivative $C_{1 \beta}$, yaw stiffness derivative $C_{\mathrm{n} \beta}$, aileron control derivative $C_{1 \delta a}$, rudder control derivative $C_{n \delta r}$ and other derivatives) even at the same flight velocity and altitude. From the comparison between state 1 and 2, we can see that as modular aircraft changes from long-endurance configuration to high-speed configuration, the wing changes from high-aspect-ratio straight one to low-aspect-ratio swept one, resulting in the variation of lateral-directional derivatives. For example, the decrease of wing area leads to the reduction of $C_{\mathrm{lp}}$. The increase of swept angle leads to the increase of $C_{1 \beta}$. The decrease of the distance between aileron and the body symmetric axis leads to the reduction of $C_{1 \delta a}$.

The lateral-directional derivatives of modular aircraft will change as the flight velocity and altitude change under the same configuration. From the comparison between state 1 and 3 we can see that when the flight velocity of the aircraft under long-endurance configuration changes from $0.2 \mathrm{Ma}$ to $0.6 \mathrm{Ma}$, and the altitude changes from $3 \mathrm{~km}$ to $10 \mathrm{~km}$, the lateral-directional derivatives also change because of the decrease of angle of attack and other factors, such as the increase of $C_{\mathrm{lp}}, C_{\mathrm{nr}}$, $C_{1 \beta}, C_{\mathrm{n} \beta}, C_{1 \delta \mathrm{a}}, C_{\mathrm{n} \delta \mathrm{r}}$.

According to the lateral-directional derivatives of modular aircraft, the lateral-directional state matrix and control matrix of typical state points can be obtained after trim and calculation. The results show the state matrix $A$ and control matrix $B$ can vary substantially at different state points.

According to lateral-directional motion equations under different configurations at various state points, the eigenvalues of modes can be solved. They are shown in Table 2.

Table 2. Eigenvalues of lateral-directional modes

\begin{tabular}{|c|c|c|c|}
\hline State & Roll mode & Spiral mode & Dutch-roll mode \\
\hline 1 & -5.215 & 0.018 & $-0.094 \pm 0.893 \mathrm{i}$ \\
\hline 2 & -1.381 & 0.003 & $-0.178 \pm 1.251 \mathrm{i}$ \\
\hline 3 & -8.175 & -0.002 & $-0.100 \pm 1.826 \mathrm{i}$ \\
\hline 4 & -2.304 & -0.012 & $-0.145 \pm 2.298 \mathrm{i}$ \\
\hline
\end{tabular}

Table 3. Specifications and flying quality of lateral-directional motion mode

\begin{tabular}{|c|c|c|c|c|c|c|}
\hline \multirow{2}{*}{ State } & \multicolumn{3}{|c|}{ Roll mode } & \multicolumn{3}{|c|}{ Spiral mode } \\
\hline & \multicolumn{2}{|c|}{$T_{\mathrm{R}} / \mathrm{s}$} & Level & \multicolumn{2}{|c|}{$T_{2 \mathrm{~s}} / \mathrm{s}$} & Level \\
\hline 1 & \multicolumn{2}{|c|}{0.19} & 1 & \multicolumn{2}{|c|}{38.3} & 1 \\
\hline 2 & \multicolumn{2}{|c|}{0.73} & 1 & \multicolumn{2}{|c|}{247.5} & 1 \\
\hline 3 & \multicolumn{2}{|l|}{0.12} & 1 & \multicolumn{2}{|l|}{-} & 1 \\
\hline 4 & \multicolumn{2}{|c|}{0.43} & 1 & \multicolumn{2}{|l|}{-} & 1 \\
\hline \multirow{2}{*}{ State } & \multicolumn{6}{|c|}{ Dutch-roll mode } \\
\hline & $\zeta_{\mathrm{d}}$ & Level & $\omega_{\text {nd }}$ & Level & $\zeta_{\mathrm{d}} \omega_{\mathrm{nd}}$ & Level \\
\hline 1 & 0.10 & 2 & 0.90 & 2 & 0.09 & 2 \\
\hline 2 & 0.14 & 2 & 1.26 & 1 & 0.18 & 2 \\
\hline 3 & 0.05 & 2 & 1.83 & 1 & 0.10 & 2 \\
\hline 4 & 0.06 & 2 & 2.30 & 1 & 0.15 & 2 \\
\hline
\end{tabular}

The specifications of lateral-directional motion mode can be solved according to the result from Table 2 and then the flying quality can also be obtained. The results are shown in Table 3.

Table 3 shows that modular aircraft lateral-directional motion modes have substantial variations at same flight velocity and altitude but under different configurations. The reason for that is the variation of lateral-directional derivatives and configuration parameters caused by the change of the configuration will lead to the difference of aircraft motion modes. For example, when modular aircraft changes from long-endurance configuration to high-speed one, the roll damping derivative $C_{\mathrm{lp}}$ will decrease, leading to the increase of the roll-mode time constant $T_{\mathrm{R}}$. The increase of the yaw damping derivative $C_{\mathrm{nr}}$ will result in the increase of the total damping value $\zeta_{\mathrm{d}} \omega_{\text {nd }}$, i.e. the product of 
the undamped natural frequency $\omega_{\text {nd }}$ and the damping ratio $\zeta_{\mathrm{d}}$ of the dutch-roll mode. From mathematical point of view, the state matrix $A$ and control matrix $B$ will vary substantially due to the change of the configuration, because the matrix elements are relative to lateral-directional derivatives, which are affected by the configuration. Furthermore, the variation of $A$ and $B$ will cause the change of eigenvalues, which determine aircraft lateral-directional motion modes.

We can also see that aircraft lateral-directional motion modes have substantial variations under same configuration but at different velocity and altitude. It is because that the change of flight velocity and altitude will also leads to difference in matrix $A$ and $B$, and finally cause different eigenvalues and lateral-directional motion modes.

Table 3 also shows that modular aircraft has good characteristics of roll mode and spiral mode but bad dutch-roll mode characteristics at most state points, without reaching the flying quality level 1 , mainly because of the insufficient damping ratio $\zeta_{\mathrm{d}}$ and total damping value $\zeta_{\mathrm{d}} \omega_{\text {nd. }}$.

Modular aircraft has different configurations and large flight envelope, which lead to the substantial variations in dynamic characteristics. The classic control method can no longer satisfy its controlling demand. The self-scheduled parameter adjusting $H_{\infty}$ robust controller must be designed to guarantee good dynamic characteristics and robustness of the aircraft under different configurations in large flight envelope.

\section{Lateral-Directional Control Law Design}

The control purpose is to achieve the tracking of modular aircraft lateral-directional command signals. The root-locus method is used to design SAS for the inner loop of the control system to guarantee the good dynamic characteristics of the system. The self-scheduled parameter adjusting $H_{\infty}$ robust controller based on LPV model is designed for the outer loop of the control system and the convex optimization theory is used to guarantee the precision tracking of lateral-directional command signals in large flight envelope. The control block diagram is shown in Fig. 4.

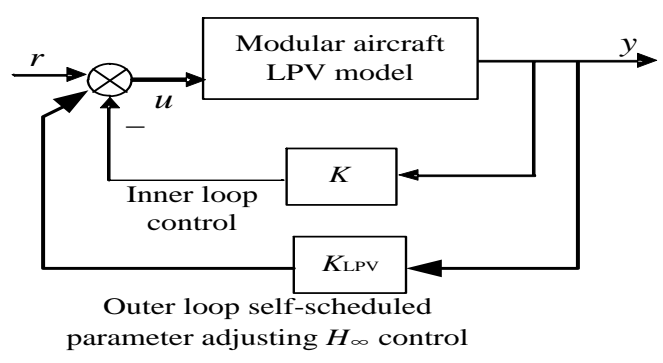

Fig. 4. Control block diagram of modular aircraft

\section{Inner Loop Controller}

The SAS control block diagram is shown in Fig. 5. The function of the SAS is to modify the dynamic characteristics of the aircraft. Body-axis roll rate is fed back to the aileron to modify the roll-subsidence mode. The yaw rate is fed back to the rudder to modify the dutch-roll mode and the sideslip angle is fed back to the rudder to ensure the lateral-directional stability.

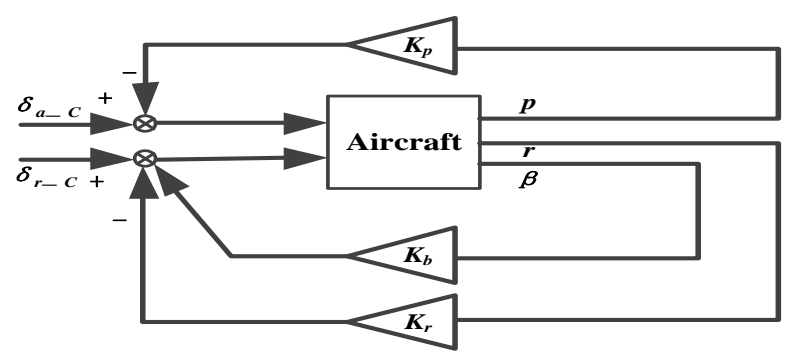

Fig. 5. SAS control block diagram 
We choose the long-endurance configuration at $0.2 \mathrm{Ma}, 3 \mathrm{~km}$ as the design point. By changing feedback parameters $K_{\mathrm{b}}, K_{\mathrm{p}}$ and $K_{\mathrm{r}}$, the pole locations can be modified. Thus we can modify aircraft lateral-directional motion modes. At last, we choose $K_{\mathrm{b}}=5.0, K_{\mathrm{p}}=-0.1$ and $K_{\mathrm{r}}=-4.0$ to be the feedback parameters, which guarantees the aircraft's good dynamic characteristics.

The eigenvalues and specifications of SAS are shown in Table 4. Compared to the flying quality criteria, the aircraft with SAS can reach flying quality level 1 at the design point.

Table 4. Eigenvalues and specifications of SAS

\begin{tabular}{|c|c|c|c|}
\hline Mode & Eigenvalue & Specification & Flying quality level \\
\hline Roll mode & -5.263 & $T_{\mathrm{R}}=0.19 \mathrm{~s}$ & 1 \\
\hline Spiral mode & 0.019 & $T_{2 \mathrm{~s}}=35 \mathrm{~s}$ & 1 \\
\hline \multirow{3}{*}{ Dutch-roll mode } & \multirow{2}{*}{$-1.248 \pm 2.370 \mathrm{i}$} & $\zeta_{\mathrm{d}}=0.464$ & 1 \\
\cline { 3 - 4 } & & $\omega_{\mathrm{nd}}=2.64 \mathrm{rad} / \mathrm{s}$ & 1 \\
\cline { 3 - 4 } & $\zeta_{\mathrm{d}} \omega_{\mathrm{nd}}=1.25 \mathrm{rad} / \mathrm{s}$ & 1 \\
\hline
\end{tabular}

We perform verifications on the SAS and find that it can also modify the aircraft dynamic characteristics at other boundary points in the flight envelope.

\section{Outer Loop Controller}

We use $H_{\infty}$ robust controller based on LPV model and convex optimization theory to solve the outer loop self-scheduled controller of the system to guarantee the aircraft's precision tracking of lateraldirectional command signals in large flight envelope. The outer loop LPV controller diagram is shown in Fig. 6.

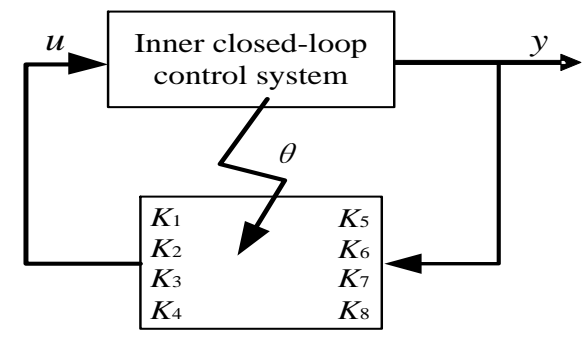

Fig. 6. Outer loop LPV controller diagram

After the design of SAS for the inner loop, we can obtain the LPV model of the closed loop system. It can be expressed as following:

$$
\left\{\begin{array}{l}
\dot{\mathbf{x}}=\mathbf{A}_{c}(\boldsymbol{\theta}) \mathbf{x}+\mathbf{B}_{2}(\boldsymbol{\theta}) \mathbf{u} \\
\mathbf{z}=\mathbf{C}_{1} \mathbf{x} \\
\mathbf{y}=\mathbf{C}_{2} \mathbf{x}
\end{array} .\right.
$$

where $\mathbf{A}_{\mathrm{c}}(\boldsymbol{\theta})$ is the augmented matrix of the system with inner loop control, $\mathbf{A}_{\mathrm{c}}=\mathbf{A}+\mathbf{B}^{*} \mathbf{K}^{*} \mathbf{C}$. $\mathbf{K}$ is the control matrix of SAS.

$$
\left\{\begin{array}{l}
\mathbf{x}=\left[\begin{array}{llllllll}
\beta & p & r & \phi & \delta_{a} & \delta_{r} & \varepsilon_{\phi} & \varepsilon_{\beta}
\end{array}\right]^{\mathrm{T}} \\
\mathbf{y}=\left[\begin{array}{llllllll}
\beta & p & r & \phi & e_{\phi} & \varepsilon_{\phi} & e_{\beta} & \varepsilon_{\beta}
\end{array}\right]^{\mathrm{T}} \\
\mathbf{z}=\left[\begin{array}{ll}
\phi & \beta
\end{array}\right]^{\mathrm{T}} \\
\mathbf{u}=\left[\begin{array}{ll}
\delta_{a} & \delta_{r}
\end{array}\right]^{\mathrm{T}}
\end{array}\right.
$$




$$
\mathbf{A}_{c}=\left[\begin{array}{cccccccc}
a_{11} & a_{12} & a_{13} & a_{14} & b_{11} & b_{12} & 0 & 0 \\
a_{21} & a_{22} & a_{23} & a_{24} & b_{21} & b_{22} & 0 & 0 \\
a_{31} & a_{32} & a_{33} & a_{34} & b_{31} & b_{32} & 0 & 0 \\
a_{41} & a_{42} & a_{43} & a_{44} & b_{41} & b_{42} & 0 & 0 \\
0 & 0 & 0 & 0 & -20.2 & 0 & 0 & 0 \\
0 & 0 & 0 & 0 & 0 & -20.2 & 0 & 0 \\
0 & 0 & 0 & -1 & 0 & 0 & 0 & 0 \\
-1 & 0 & 0 & 0 & 0 & 0 & 0 & 0
\end{array}\right] .
$$

where $a$ and $b$ are matrix elements which have function relationship with aircraft flight velocity, altitude and the configuration.

$$
\mathbf{B}_{2}=\left[\begin{array}{cc}
0 & 0 \\
0 & 0 \\
0 & 0 \\
0 & 0 \\
20.2 & 0 \\
0 & 20.2 \\
0 & 0 \\
0 & 0
\end{array}\right], \mathbf{C}_{1}=\left[\begin{array}{cc}
1 & 0 \\
0 & 0 \\
0 & 0 \\
0 & 1 \\
0 & 0 \\
0 & 0 \\
0 & 0 \\
0 & 0
\end{array}\right], \mathbf{C}_{2}=\left[\begin{array}{cccccccc}
1 & 0 & 0 & 0 & 0 & 0 & 0 & 0 \\
0 & 1 & 0 & 0 & 0 & 0 & 0 & 0 \\
0 & 0 & 1 & 0 & 0 & 0 & 0 & 0 \\
0 & 0 & 0 & 1 & 0 & 0 & 0 & 0 \\
0 & 0 & 0 & -1 & 0 & 0 & 0 & 0 \\
0 & 0 & 0 & 0 & 0 & 0 & 1 & 0 \\
-1 & 0 & 0 & 0 & 0 & 0 & 0 & 0 \\
0 & 0 & 0 & 0 & 0 & 0 & 0 & 1
\end{array}\right] .
$$

The designed controller can be expressed as the following form:

$$
\mathbf{u}=\mathbf{K}(\boldsymbol{\theta}) \mathbf{y} \text {. }
$$

The control objective is to realize the tracking of sideslip angle $\beta$ and roll angle $\varphi$.

The boundary state points of modular aircraft flight envelope are four state points, which can be expressed in form of flight velocity and altitude as $(0.2 \mathrm{Ma}, 3 \mathrm{~km}),(0.2 \mathrm{Ma}, 10 \mathrm{~km}),(0.6 \mathrm{Ma}, 3 \mathrm{~km})$ and $(0.6 \mathrm{Ma}, 10 \mathrm{~km})$. At each boundary state points, the modular aircraft will have two configurations as long-endurance configuration and high-speed configuration.

To solve the outer loop controller, the matrix elements in A and B at boundary points must be obtained at first. Thus, we can determine the range of matrix elements in the flight envelope. When we use convex optimization theory to solve the LPV controller, the computation load will increase as the number of variable elements in matrix $\operatorname{Ac}(\theta)$ increase. In order to reduce the computation load, we only take into consideration matrix elements that have a wide variation range. Those elements that have minor variation range will be considered as constants.

According to the modular aircraft LPV model we can conclude that matrix elements a22, a31 and b32 in $\operatorname{Ac}(\theta)$ have a wide variation range in the flight envelope. The roll damping characteristic is reflected by a22. The sideslip stiffness characteristic is reflected by a31. The yaw damping characteristic is reflected by b32. These three matrix elements will have a substantial effect on the design of controller. Therefore, we choose the three elements as scheduling parameters for LPV controller. Other matrix elements that have minor variation ranges and less effect on the controller are considered as constants:

$$
\begin{aligned}
& \left\{\begin{array}{l}
a_{22} \subset\left[\begin{array}{ll}
a_{22 \min } & a_{22 \max }
\end{array}\right]=\left[\begin{array}{ll}
-18.1 & -1.6
\end{array}\right] \\
a_{31} \subset\left[\begin{array}{ll}
a_{31 \min } & a_{31 \max }
\end{array}\right]=\left[\begin{array}{ll}
2.2 & 16.6
\end{array}\right] \\
b_{32} \subset\left[\begin{array}{ll}
b_{32 \min } & b_{32 \max }
\end{array}\right]=\left[\begin{array}{ll}
-11.7 & -1.3
\end{array}\right]
\end{array} .\right. \\
& \boldsymbol{\theta} \in \operatorname{Co}\left\{\boldsymbol{\theta}_{i}, i=1,2,3,4,5,6,7,8\right\} .
\end{aligned}
$$

where 


$$
\begin{aligned}
& \boldsymbol{\theta}_{1}=\left[\begin{array}{lll}
a_{22 \min } & a_{31 \min } & b_{32 \min }
\end{array}\right]^{T}, \boldsymbol{\theta}_{2}=\left[\begin{array}{lll}
a_{22 \max } & a_{31 \min } & b_{32 \min }
\end{array}\right]^{T}, \\
& \boldsymbol{\theta}_{3}=\left[\begin{array}{lll}
a_{22 \min } & a_{31 \max } & b_{32 \min }
\end{array}\right]^{T}, \boldsymbol{\theta}_{4}=\left[\begin{array}{lll}
a_{22 \min } & a_{31 \min } & b_{32 \max }
\end{array}\right]^{T}, \\
& \boldsymbol{\theta}_{5}=\left[\begin{array}{llll}
a_{22 \max } & a_{31 \max } & b_{32 \min }
\end{array}\right]^{T}, \boldsymbol{\theta}_{6}=\left[\begin{array}{lll}
a_{22 \max } & a_{31 \min } & b_{32 \max }
\end{array}\right]^{T}, \\
& \boldsymbol{\theta}_{7}=\left[\begin{array}{llll}
a_{22 \min } & a_{31 \max } & b_{32 \max }
\end{array}\right]^{T}, \boldsymbol{\theta}_{8}=\left[\begin{array}{lll}
a_{22 \max } & a_{31 \max } & b_{32 \max }
\end{array}\right]^{T} .
\end{aligned}
$$

Therefore, $\mathbf{A}_{c}(\boldsymbol{\theta})$ is included in a convex polyhedron, which can be expressed as following:

$$
\mathbf{A}_{c}(\boldsymbol{\theta}) \in \operatorname{Co}\left\{\mathbf{A}_{i}, i=1,2,3,4,5,6,7,8\right\} .
$$

where $\mathbf{A}_{\mathrm{i}}$ is the mapping matrix of eight boundary points $\boldsymbol{\theta}_{1}, \boldsymbol{\theta}_{2}, \boldsymbol{\theta}_{3}, \boldsymbol{\theta}_{4}, \boldsymbol{\theta}_{5}, \boldsymbol{\theta}_{6}, \boldsymbol{\theta}_{7}, \boldsymbol{\theta}_{8}$ in the parameter set.

The controller of each of the eight boundary points can be solved by using MATLAB LMI toolbox. For state matrix of a certain flight state in the envelope, the variable parameter is $\boldsymbol{\theta}=\left[a_{22}\right.$ $\left.\begin{array}{ll}a_{31} & b_{32}\end{array}\right]^{T}$. The convex composition of it can be expressed as following:

$$
\left[\begin{array}{l}
a_{22} \\
a_{31} \\
b_{32}
\end{array}\right]=\sum_{i=1}^{8} \alpha_{i} \boldsymbol{\theta}_{i}\left(\alpha_{i} \geq 0, \sum_{i=1}^{r} \alpha_{i}=1\right) .
$$

Thus, we obtain the LPV controller of the certain state point:

$$
\left[\begin{array}{ll}
\mathbf{A}_{K}(\boldsymbol{\theta}) & \mathbf{B}_{K}(\boldsymbol{\theta}) \\
\mathbf{C}_{K}(\boldsymbol{\theta}) & \mathbf{D}_{K}(\boldsymbol{\theta})
\end{array}\right]=\sum_{i=1}^{8} \alpha_{i}(t)\left[\begin{array}{ll}
\mathbf{A}_{K i} & \mathbf{B}_{K i} \\
\mathbf{C}_{K i} & \mathbf{D}_{K i}
\end{array}\right] .
$$

We can obtain the modular aircraft lateral-directional LPV controller at a certain state point in the flight envelope by substituting the results of (15) into (5).

\section{Simulation Result}

The closed loop system consists of inner and outer loop controller is simulated at typical state points. The simulation results are shown in Fig. 7 and Figure 8. (The simulation of long-endurance configuration is performed at $0.2 \mathrm{Ma}, 3 \mathrm{~km}$ and the simulation of high-speed configuration is performed at $0.6 \mathrm{Ma}, 10 \mathrm{~km}$.)
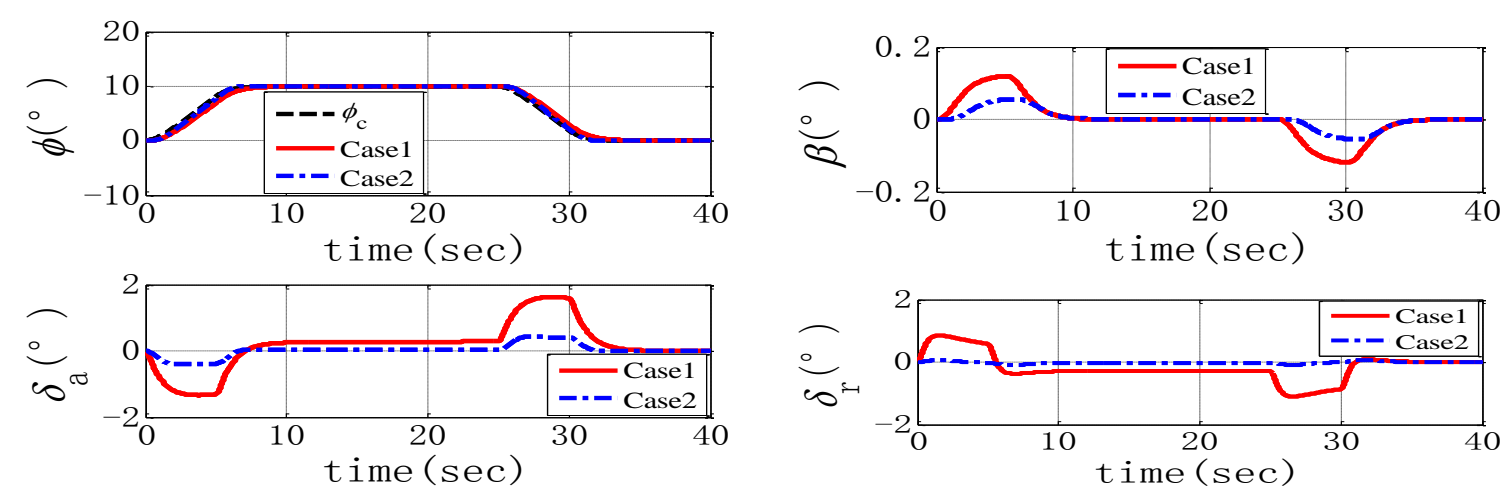

Fig. 7. Responses to command tracking of roll angle (Case 1 refers to the long-endurance configuration at $0.2 \mathrm{Ma}, 3 \mathrm{~km}$. Case 2 refers to the high-speed configuration at $0.6 \mathrm{Ma}, 10 \mathrm{~km}$.) 

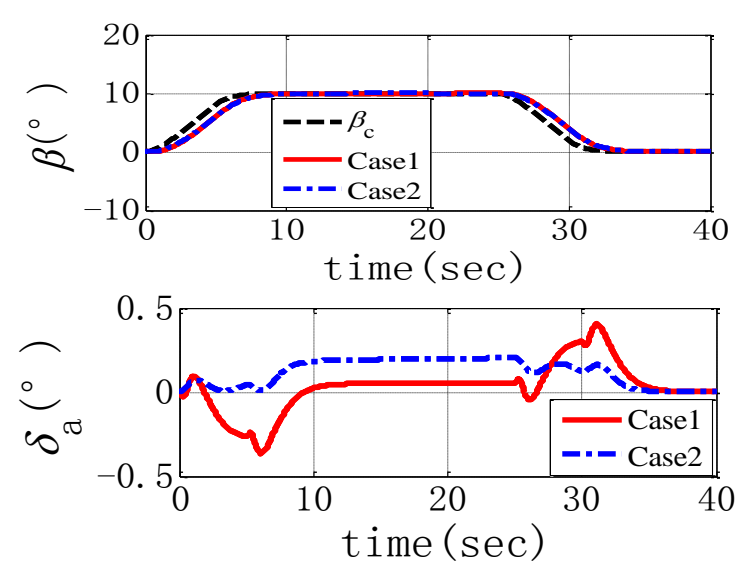
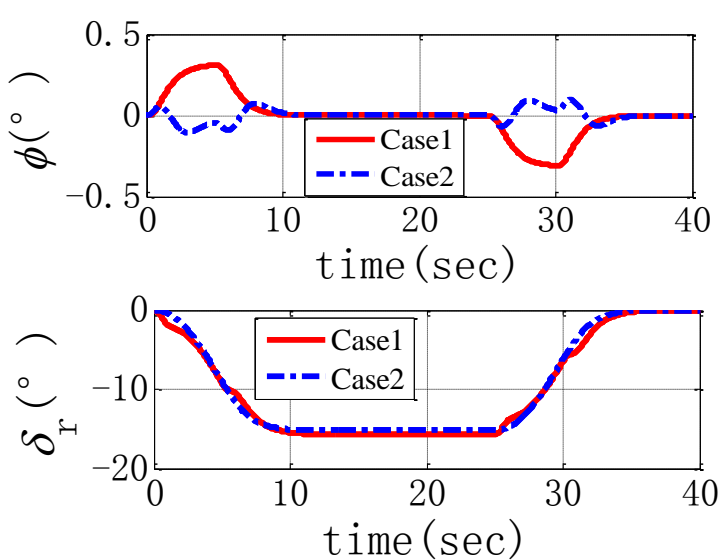

Figure 8. Responses to command tracking of sideslip angle (Case 1 refers to the long-endurance configuration at $0.2 \mathrm{Ma}, 3 \mathrm{~km}$. Case 2 refers to the high-speed configuration at $0.6 \mathrm{Ma}, 10 \mathrm{~km}$.)

The simulation results show that the closed loop system can achieve precision tracking of command signals of roll angle and sideslip angle under different configurations. When the aircraft is tracking roll angle command signal, the sideslip angle keeps as a very low value, which indicates the closed loop system realize a lateral-directional decoupling control. The deflexion of aileron and rudder is in the valid range. As the result shows, the self-scheduled parameter adjusting $H_{\infty}$ robust controller based on LPV model can guarantee the good control for modular aircraft lateraldirectional motion in large flight envelope.

\section{Summary}

We build the lateral-directional LPV model of modular aircraft in large flight envelope. The validity of the LPV model is verified by comparison with the aircraft nonlinear model. Then the modular aircraft lateral-directional dynamic characteristics under different configurations in large flight envelope are studied. We present the self-scheduled parameter adjusting $H_{\infty}$ robust control method based on the LPV model of the aircraft. The inner loop of the control system is SAS based on rootlocus method, and the $H_{\infty}$ robust control method based on LPV model is used to build the outer loop of the control system, using convex optimization theory to solve the self-scheduled parameter adjusting controller to guarantee the precision tracking of modular aircraft lateral-directional command signals. At last, the dynamic simulation is done. The results show the self-scheduled parameter adjusting $H_{\infty}$ robust controller based on LPV model of modular aircraft could adjust its parameter according to the change of modular aircraft's configuration, flight velocity and height, and guarantee good control effects for the aircraft under different configurations in large flight envelope.

\section{References}

1. Spillman, M.S, Robust longitudinal flight control design using linear parameter varying feedback, J. JGCD. 23, 101-108 (2000).

2. Biannic J, Apkarian P, Garrard W.L, Parameter varying control of a high-performance aircraft, J. J GCD. 20, 225-231 (1997).

3. Natesan K, Gu D, Postlethwaite I, Design of static $H_{\infty}$ linear parameter varying controllers for unmanned aircraft, J. JGCD. 30, 1822-1827 (2007).

4. Li Wenqiang, Ma Jianjun, Li Peng, Zheng Zhiqiang. Variable gain control based on LPV and the application in aerospace, J. AW. 2, 8-12 (2009). (in Chinese)

5. Apakarian P, Gahinet P. A convex characterization of gain-scheduled $H_{\infty}$ controllers, J. IEEE TAC. 40, 853-864 (1995). 
6. B. Etkin. Dynamic of Atmospheric Flight, first ed., Science Publishing Company, Beijing (1979). (in Chinese)

7. Fang Zhenping. Flight Dynamics of Aerospace Flight Vehicle, second ed., Press of BUAA, Beijing (2005). (in Chinese)

8. Yu Li. Robust Control: Linear Matrix Inequality Approach, first ed., Tsinghua University Press, Beijing (2002). (in Chinese)

9. Apkarian P, Gahinet P, Becker G, Self-scheduled $H_{\infty}$ control of linear parameter varying systems: a design example, J. A. 31, 1251-1261 (1995).

10. Kumar A, Andersen M.R. A comparison of LPV modelling techniques for aircraft control. AP, 2000-4458 (2000).

11. Xiao Hua, Wang Lixin. Multivariable robust lateral-directional control design for W-shaped tailless aircraft, J. JBAA. 33, 1256-1260 (2007). (in Chinese)

12. Marcos A, Balas G.J, Development of linear-parameter-varying models for aircraft, J. JGCD, 27, 218-228 (2004).

13. Rugh W.J, Shamma J.S, Research on gain-scheduled autopilot, J. JGCD.

14. Packard A, Gain-scheduling via linear fractional transformations, J. SCL, 22, 79-92 (1994) 